\title{
SOBRE OS TIPOS DE LEPIDOPTERA DEPOSITADOS EM MUSEUS BRASILEIROS. XXIII. PAPILIONIDAE, PIERIDAE E NYMPHALIDAE (MORPHINAE, NYMPHALINAE) DESCRITOS POR J.F. ZIKÁN (SUPLEMENTO) E APATELODIDAE DESCRITOS POR M. DRAUDT ${ }^{1}$
}

\author{
Olaf H.H. Mielke ${ }^{2}$ \\ Mirna M. Casagrande ${ }^{2}$
}

\begin{abstract}
ABOUT THE TYPES OF LEPIDOPTERA DEPOSITATED IN BRAZILIAN MUSEUMS. XXIII. PApilionidae, Pieridae and Nymphalidae (Morphinae, Nymphalinae) desCRIBED BY J.F. ZiKÁN (SUPPLEMENT) AND APATELODIDAE DESCRIBED BY M. DRAUDT. A supplementary list of the Papilionidae, Pieridae, Nymphalinae (Morphinae, Nymphalinae) described by J.F. Zikán, with the repositories of their types, and informes about the Apatelodidae described by M. Draudt, from the Zikán collection, are presented. Lectotypes are designated for Papilio agathosilaus Zikán, 1938; Phoebis trite race chryseida Zikán, 1940; Phoebis trite race chryseida form ismene Zikán, 1940; and Morpho hercules form irideus Zikán, 1935.
\end{abstract}

KEY WORDS. Lepidoptera, types, brasilian museums.

Em 1985 inicou-se através da Revista Brasileira de Entomologia, uma série de vinte e dois artigos sobre os tipos de Lepidoptera depositados em museus brasileiros, com o objetivo de esclarecer a sua localização. Tão logo concluiu-se o artigo de número XXII (MIELKE \& CASAGRANDE 1991), já tínha-se mais dados e sugestões de colegas sobre a necessidade de novas buscas em algumas coleções brasileiras, pois supunha-se que mais "tipos" pudessem ser encontrados, o que realmente ocorreu.

A metodologia segue MielKe \& CASAGRANDE (1985).

Neste artigo são apresentadas notas complementares aos trabalhos de MIELKE \& CASAGRANDE (1987a,b,c,d) sobre os tipos de Lepidoptera descritos por J.F. Zikán e informações sobre as espécies de Apatelodidae descritas por M. Draudt e citadas na descrição original como depositadas na Coleção J.F. Zikán, atualmente no Instituto Oswaldo Cruz, Rio de Janeiro.

\section{Tipos de espécies descritas por J.F. Zikán}

\section{PAPILIONIDAE}

Papilio agathosilaus Zikán, 1938: 281, 391; figuras de valvas nº 6, fig. 1 (genitália macho); 1 macho, agosto e 1 fêmea, novembro, Alto Tijuca, Rio de Janeiro, Estado do Rio de Janeiro, Brasil, P. Gagarin leg.; coleção Zikán.

Dos dois síntipos encontrados, designa-se como lectótipo (Fig. 1) o macho de 2-VIII-1934, n 20.667, e paralectótipo a fêmea de 27-XI-1931, nº 20.668, ambos no Instituto Oswaldo Cruz, Rio de Janeiro.

1) Contribuição número 1069 do Departamento de Zoologia, Universidade Federal do Paraná.

2) Departamento de Zoologia, Universidade Federal do Paraná. Caixa Postal 19020, 81531-990 Curitiba, Paraná, Brasil. Bolsista do CNPq. 
Os dois exemplares já foram assim rotulados em 1993 pelo Dr. Gerardo Lamas (Peru).

Sinônima de Protesilaus travassosi (D’Almeida, 1938)

\section{PIERIDAE}

Phoebis trite raça chryseida Zikán, 1940: 158; macho, Espírito Santo, Minas Gerais, Rio de Janeiro e São Paulo, [Brasil].

Somente um síntipo foi encontrado e é designado como lectótipo (Fig. 2); macho 21-V-1924, Itatiaia, estado do Rio de Janeiro, Brasil, J.F. Zikán leg., no 21.123; Instituto Oswaldo Cruz, Rio de Janeiro.

Sinônima de Rhabdodryas trite banksi (Breyer, 1939)

Phoebis trite raça chryseida f. ismene Zikán, 1940: 158, fêmea [Itatiaia, Estado do

Rio de Janeiro, Brasil].

Dos dois síntipos encontrados, designa-se como lectótipo (Fig. 3) a fêmea de 23-V-1924, Itatiaia, estado do Rio de Janeiro, Brasil, J.F. Zikán leg., n² 21.139 e como paralectótipo a fêmea de $19-\mathrm{V}-1928$, da mesma procedência, $\mathrm{n}^{\circ} 21.133$, ambos no Instituto Oswaldo Cruz, Rio de Janeiro.

Sinônima de Rhabdodryas trite banksi (Breyer, 1939)

\section{NYMPHALIDAE}

\section{Morphinae}

Morpho hercules f. irideus Zikán, 1935: 140, macho, fêmea, Itatiaia, Estado do Rio de Janeiro e Mar de Espanha, Minas Gerais, [Brasil].

Dos dois síntipos encontrados, designa-se como lectótipo (Fig. 4) o macho de 22-II-1935, Itatiaia, Estado do Rio de Janeiro, 700 m, J.F. Zikán leg., n 30.729 , e como paralectótipo o macho de 6-II-1935, da mesma procedência, $n^{\circ} 30.786$, ambos no Intstituto Oswaldo Cruz, Rio de Janeiro.

Sinônima de Iphimedeia hercules (Dalman, 1823).

\section{Nymphalinae}

Pyrameis myrinna ab. merlinde Zikán, 1937: 386, pl. 9, fig. 1 (fềmea dorsal); 1 fêmea [holótipo] 1-IV-1934, Itatiaia, Estado do Rio de Janeiro, [Brasil, 700 m, J.F. Zikán leg.].

O holótipo (Fig. 5), n² 26.228, está no Instituto Oswaldo Cruz, Rio de Janeiro. Sinônima de Vanessa myrinna (Doubleday, 1849).

\section{Tipos de espécies descritas por M. Draudt}

\section{APATELODIDAE}

Os Apatelodidae descritos por Draudt e citados nas descrições originais como estando na Coleção J.F. Zikán, atualmente no Instituto Oswaldo Cruz, Rio de Janeiro, não foram encontrados. Provavelmente J.F. Zikán enviou um exemplar de cada uma das espécies para o Dr. M. Draudt que não os retornou. Não foi possível

Figs 1-3. (1) Papilio agathosilaus Zikán, lectótipo macho, faces dorsal e ventral; (2) Phoebis trite raça chryseida Zikán, lectótipo macho, faces dorsal e ventral; (3) Phoebis trite raça chryseida f. ismene Zikán, lectótipo macho, faces dorsal e ventral. 

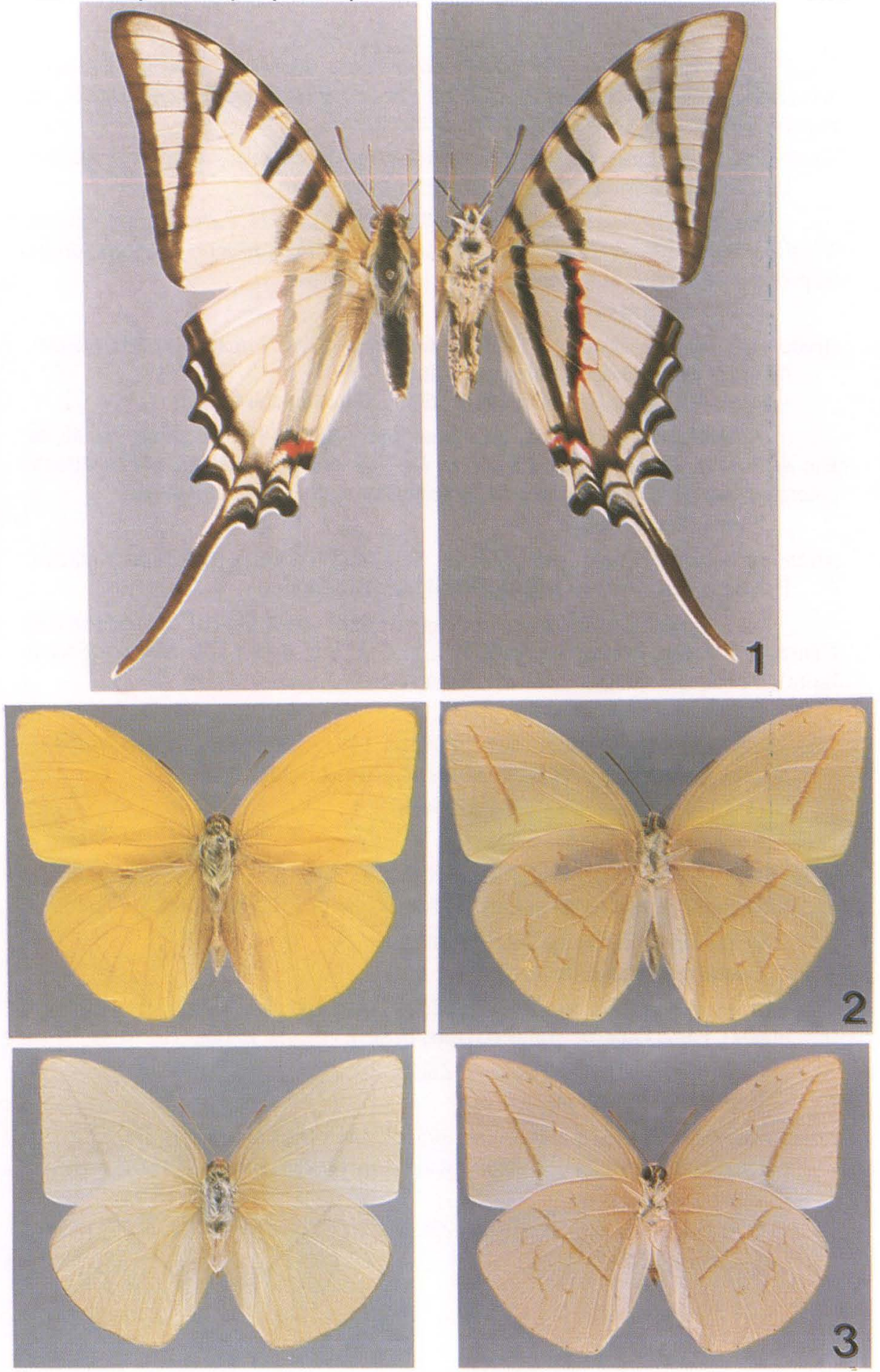

Revta bras. Zool. 15 (4): 1069 - 1074, 1998 
localizá-los e supomos que se ainda existirem devem ser procurados em museus da Alemanha. Veja comentários em cada uma das espécies a seguir mencionadas. As interpretações sistemáticas seguem BECKER (1996).

Apatelodes zikani Draudt, 1929: 695, pl. 140b (macho dorsal); [holó]tipo, 1 macho, Passa Quatro, Minas [Gerais], Brasil; coleção Zikán.

Na coleção Zikán há um exemplar "tipo" de 5-X-1921, Fazenda dos Campos, Virgínia, Sul de Minas Gerais, 1500 m, Zikán leg. Não pode ser o holótipo porque as procedências não coincidem.

É uma espécie válida.

Apatelodes thinaha Draudt, VII-1929: 695; [holó]tipo, 1 macho, Passa Quatro, Minas [Gerais, Brasil]; coleção Zikán.

Apatelodes tinaha [sic]; Draudt, 1929, pl. 140c (macho dorsal).

Na coleção Zikán há um exemplar "tipo" de 3-X-1929, Itatiaia, estado do Rio de Janeiro, Brasil, 700m, J.F. Zikán leg. Não pode ser holótipo pois a data de coleta é posterior à publicação e as procedências também não coincidem.

É uma espécie válida.

Apatelodes cessita Draudt, 1929: 698, pl. 140f (macho dorsal); [holó]tipo, 1 macho, Passa Quatro, Minas [Gerais], Brasil; coleção Zikán.

Na coleção Zikán há um exemplar "tipo" de 7-VI-1917, Fazenda dos Campos, Virgínia, Minas Gerais, Brasil, 1500 m, J.F. Zikán, leg. Não pode ser o holótipo porque as procedências não coincidem.

É uma espécie válida.

Apatelodes xanthapex Draudt, 1929: 699, pl. 141a (macho dorsal); [holó]tipo, 1 macho, Passa Quatro, Minas [Gerais, Brasil]; coleção Zikán.

O holótipo não está na coleção Zikán.

É uma espécie válida.

Apatelodes concerpta f. fucosa Draudt, 1929: 700, pl. 141a (macho dorsal); [holó]tipo, 1 macho, Passa Quatro, Minas [Gerais, Brasil]; coleção Zikán.

O holótipo não está na coleção Zikán.

É uma espécie válida.

Thelosia postflavida Draudt, 1929: 702, pl. 141d (macho dorsal); [holó]tipo, 1 macho, Passa Quatro, Minas [Gerais, Brasil]; coleção Zikán.

O holótipo não está na coleção Zikán.

É uma espécie válida.

Olceclostera basifusca Draudt, 1929: 705, pl. 14lh (macho dorsal); [holó]tipo, 1 macho, Campo Bello [Itatiaia, Estado do] Rio de Janeiro, [Brasil]; coleção Zikán.

O holótipo não está na coleção Zikán.

É uma espécie válida. 

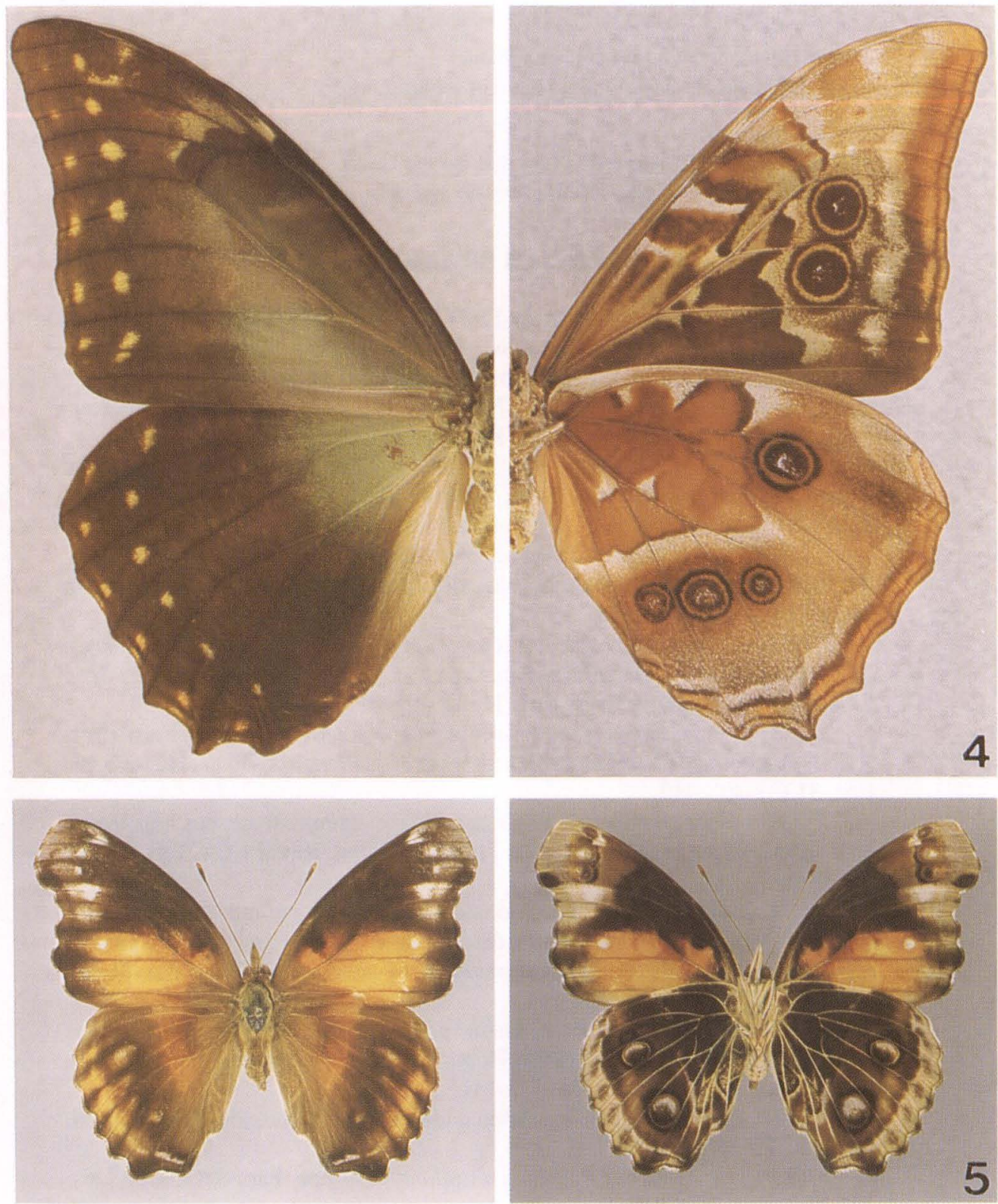

Figs 4-5. (4) Morpho hercules f. irideus Zikán, lectótipo macho, faces dorsal e ventral; (5) Pyrameis myrinna ab. merlinde Zikán, holótipo fêmea, faces dorsal e ventral. 
Colabata illauta Draudt, 1929: 708, pl. 142c [não existe]; tipos macho e fêmea, Passa Quatro, Minas [Gerais, Brasil]; coleção Zikán.

Os tipos não estão na coleção Zikán.

É uma espécie de Ephoria Herrich-Schäffer, 1855.

AGRADECIMENTOS. Agradecemos aos Drs Gerardo Lamas, Lima, Peru e Vitor Becker, Brasília, Distrito Federal, pelas informações sobre os tipos.

\section{REFERÊNCIAS BIBLIOGRÁFICAS}

BECKER, V.O. 1996. Apatelodidae, p.13-17. In: J.B. HEPPNER (Ed.). Atlas of Neotropical Lepidoptera. Checklist: Part 4B Drepanoidea - Bombycoidea - Sphingoidea. Gainesville, Scientific Publishers, L+87p.

DraudT, M. 1929. Apatelodes, p.695-700. In: A. SEITZ (Ed.) Gross-Schmetterlinge der Erde 6. Stuttgart, Alfred Kernen, 1452p.

Mielke, O.H.H. \& M.M. Casagrande. 1985. Sobre os tipos de Lepidoptera depositados em museus brasileiros. I. Papilionidae, Riodinidae, Hesperiidae e Arctiidae, descritos por R.F. D'Almeida. Revta bras. Ent. 29 (2): 317-320. . 1987a. Sobre os tipos de Lepidoptera depositados em museus brasileiros. XI. Nymphalidae (Nymphalinae) descritos por José Francisco Zikán. Revta bras. Ent. 31 (3): 399-403.

1987b. Sobre os tipos de Lepidoptera depositados em museus brasileiros. XII. Nymphalidae (Danainae, Satyrinae e Charaxinae) descritos por José Francisco Zikán. Revta bras. Ent. 31 (3): 404-407.

- $1987 \mathrm{c}$. Sobre os tipos de Lepidoptera depositados em museus brasileiros. XIII. Nymphalidae (Ithomiinae) descritos por José Francisco Zikán. Revta bras. Ent. 31 (3): 408-413.

. 1987d. Sobre os tipos de Lepidoptera depositados em museus brasileiros. XIV. Riodinidae descritos por José Francisco Zikán. Revta bras. Ent. 31 (3): 414-419.

1991. Sobre os tipos de Lepidoptera depositados em museus brasileiros.

XXII. Nymphalidae (Charaxinae) descritos por A. H. Fassl (Suplemento) e Nymphalidae (Charaxinae), Papilionidae e Lycaenidae por M. Rosa. Revta bras. Ent. 35 (1): 29-34.

ZIKÁN, J.F. 1935. Morpho hercules Dalm. und richardus Fruhst. ein auffälliges Beispiel von Parallelismus bei Lepidopteren der neotropischen Fauna. Dtsch. Ent. Ztschr. Iris, Dresden, 49: 133-141.

1937. Neue Nymphaliden-Arten und-Formen aus Brasilien. Ent. Rdsch. 54 (31): 385-387.

. 1938. Die Arten der Papilio protesilaus - Gruppe. Ent. Rdsch. 55 (25): 281-286; (34): 299-393.

. 1940. Beschreibungen neuer neotropischer Papilionidae, Pieridae,

Danaide und Satyridae. Ent. Ztschr., Stuttgart, 54 (20): 154-159.

Recebido em 10.VIII.1997; aceito em 27.XI.1998 